\title{
Twin Relationships in Ingots of Germanium
}

\author{
by W. C. Ellis
}

$I^{1}$

$\mathrm{N}$ an ingot of germanium solidified progressively from the bottom by a method' devised by J. $\mathrm{H}$. Scaff and H. C. Theuerer, examination discloses ex-

1 4 C Torrey and C. A. Whitmer, Crystal Rectifers. p. 364 1948. New York. McGraw-HHll Pub. Co.

tensive twin relationships. A slice taken about midway in a cylindrical ingot approximately $3 / 4$ in. in diameter and $11 / 2$ in. in length, after etching and polishing to delineate different orientations, is shown in fig. 1 a. The orientations of the regions designated with letters were determined by Laue X-ray backreflection methods and plotted on stereograms. The orientation relationships of the designated components were determined by comparing the values for the nine angles between the three respective cube poles of each orientation with the values calculated in a formal treatment of octahedral multiple twinning in cubic crystals. Germanium, diamond cubic in structure, has the octahedral twinning habit.

I am indebted to K. H. Storks for the calculation of the angular relationships of cube poles in octahedral twinning to four orders. The set of values for the nine angles is distinctive of the order of twinning. Confirmation for a multiple twin relationship is obtained from the determined indices of the mirror planes. These exist for all orders of twinning through the third and for some of the fourth.* If the orientations are not those for twinning, then the nine angles for the cube poles do not correspond in value and distribution to those formally calculated. Since all nine angles must correspond in values and distribution of values to the calculated ones, the Laue method with a precision of one or two degrees is adequate to establish or deny with confidence the existence of multiple twins. Regions of the same orientation can also frequently be identified by the optical reflections. For example, the narrow twin bands in $A$ at 2:00 o'clock have orientation $B_{1}$.

The interesting twin relationships shown schematically in fig. $1 b$ were found for the section in fig. 1a. Most of this slice was made up of orientation, $A$, appearing in widely separated regions. Of the four possible first order twins of $A$, three were present in this slice and are designated by $B$ with subscripts. Four second order twins of $A$ are designated by $C$ with subscripts. It is worth emphasizing that two of these, $C_{4}$ and $C_{5}$, are second orders of $A$ through the first order missing in this section. Two regions, designated by $D$ with subscripts are third order twins of $A . D_{2}$ is a sixth order twin of $D_{4}$. All orientations found were ascribable to multiple twinning, as if the orientations in this section originated earlier in solidification from a single nucleus.

The presence of extensive multiple twinning to-

W. C. ELLIS, Member AIME, is Member of Technical Staff, Bell Telephone Laboratories, Inc., New York, New York.

Technical Note No. 37 E. Manuscript received March 1,1950 .

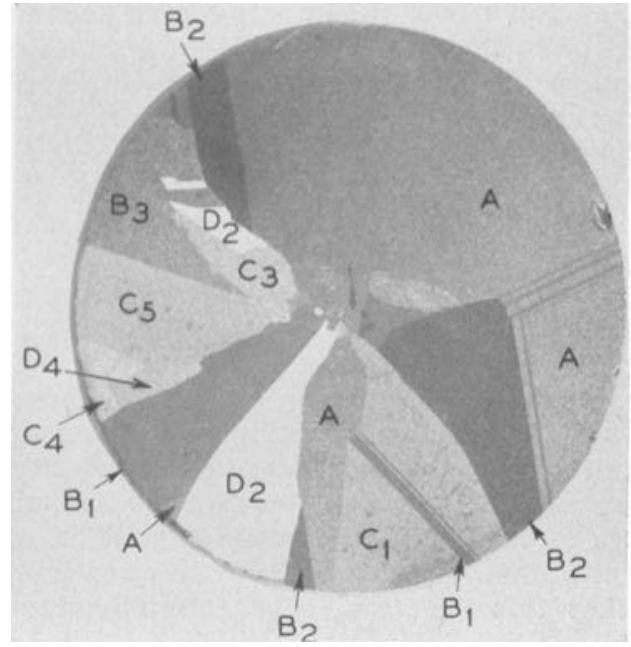

a

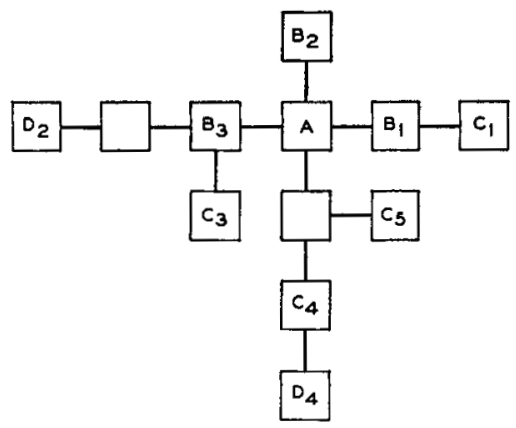

$b$

Fig. 1a-Etched transverse section of germanium ingot showing regions of different orientations. X3.5. Reduced approximately one third in reproduction.

Fig. 1b-Schematic representation of twin relationships in section shown in fig. 1a.

gether with the absence of other orientations appears to be a characteristic result in many instances of solidification of germanium. Fertile conditions for twinning probably exist in the array of atoms in the diamond cubic structure. The high order twins in the section shown, it is reasonable to presume, are the result of progressive octahedral twinning through several generations in the material freezing earlier. Some of these generations do not appear in this section, it is suggested, because their orientations with respect to the growth direction were unfavorable for survival. The extensive twinning is probably promoted during solidification by constraint imposed by volume increase, and to an irregular growth front. Twinning may also be due, in part, to the attempt of the solidifying region to attain the orientation most favorable to the growth direction (maximum thermal gradient). The nucleus for a twinned orientation could have its origin in a "stacking fault" on the close-packed octahedral plane.

Further work is being done in which the course of twinning throughout an ingot is being studied. The results appear to be important in the general theory of solidification. 